\title{
Effect of the National Resident Assessment Instrument on Selected Health Conditions and Problems
}

\author{
Brant E. Fries, $P h D,{ }^{*}$ Catherine Hawes, $P h D,{ }^{\dagger} J o b n$ N. Morris, $P h D,{ }^{\neq}$ \\ Charles D. Phillips, $\mathrm{PhD}, \mathrm{MPH},{ }^{\mathrm{S}}$ Vince Mor, $\mathrm{PhD}$," and Pil S. Park, $\mathrm{PhD}{ }^{\dagger t}$
}

OBJECTIVE: To evaluate the effect of the implementation of the National Resident Assessment Instrument (RAI) system on selected conditions representing outcomes for nursing home residents.

DESIGN: Quasi-experimental, pre-/post-design, with assessments at baseline and 6-month follow-up.

SAMPLE: Two thousand one hundred twenty-eight residents from 268 nursing homes in 10 states before RAI implementation, and 2,088 from 254 of the same nursing homes after implementation.

MEASURES: From the full RAI Minimum Data Set, measures of dehydration, falls, decubitus, vision problems, stasis ulcer, pain, dental status (poor teeth), and malnutrition were examined at baseline and 6 months later. Poor nutrition was evaluated using a body mass index score below 20 and vision using a 4-level scale; other conditions were represented by their presence or absence. Decline and improvement were computed as the changes in level between baseline and follow-up, limiting the sample to those who could manifest each such change.

MAIN RESULTS: Of eight health conditions representing poorer health status, dehydration and stasis ulcer had significantly lower prevalence after the implementation of the RAI (1993) compared with 1990. At the same time, there was an increase in the prevalence of daily pain. Fewer residents declined over 6 months in nutrition and vision after implementation. Although for these two conditions there were also significantly reduced rates of improvement, the net was an overall reduction in the 6-month rate of decline for all residents. Pain also demonstrated a decline in the postimplementation rate of improvement. The combined eight conditions showed reductions in the rates of both decline and improvement.

From the "Institute of Gerontology and School of Public Health, University of Michigan; and Ann Arbor VA Medical Center, Ann Arbor, Michigan; Program on Aging and Long-Term Care, Research Triangle Institute, Research Triangle Park, North Carolina; †Social and Gerontological Research Institute, Hebrew Rehabilitation Center for Aged, Boston, Massachusetts; $S_{\text {Myers Research Insti- }}$ tute, Menorah Park Center for the Aging, Beachwood, Ohio; llCenter for Gerontology and Health Care Research, Brown University, Providence, Rhode Island; tt Institute of Gerontology, University of Michigan, Ann Arbor, Michigan.

This research was supported by the Health Standards and Quality Bureau of the Health Care Financing Administration, under Contract 500-88-0055. The opinions expressed here are those of the authors and do not represent official policy of the funding agency. Portions of this paper were presented at the Annual Meeting of the Gerontological Society of American in Atlanta, November 1994.

Address correspondence to Brant E. Fries, PhD, Institute of Gerontology, University of Michigan, 300 North Ingalls, Ann Arbor, Ml 48109-2007.
CONCLUSIONS: Several outcomes for nursing home residents improved after implementation of the RAI. Of the four conditions for which there are significant declines in prevalence or outcome changes, three are specifically addressed in the care planning guidelines incorporated the RAI system (all except stasis ulcer, although there is a RAP for decubitus ulcer). Pain, the only other condition with a significant result - an increase in baseline prevalence - also has no RAP. Although the changes might be ascribed otherwise, they support the premise that the RAI has directly contributed to improved outcomes for nursing home residents. J Am Geriatr Soc 45:994-1001, 1997.

$T$ The nursing home industry in the United States has been faced this decade with numerous challenges brought on by the reforms mandated in the Omnibus Budget Reconciliation Act of 1987 (OBRA-87). Among the several provisions of this law to improve quality of care in nursing homes was the requirement that the federal government develop, implement, and then evaluate a comprehensive system to assess residents. In this article we consider the effect of the implementation of the National Resident Assessment Instrument (RAI) ${ }^{1}$ on specific residents' health and medical conditions. For the selected conditions, we examined whether the RAI affected either the prevalence or incidence of the condition or the likelihood that a discovered condition would be resolved.

For editorial comment, see pp 975, 1025, 1027

By October 1991, the RAI was implemented in all US nursing homes as a requirement of the Conditions of Participation for the Medicare and Medicaid programs. As a result, virtually every nursing home completes these assessments. Every resident must be assessed using the RAI's Minimum Data Set (MDS) and have individualized care planning based on the information contained in the RAI Resident Assessment Protocols (RAPs). ${ }^{2}$ The 18 RAPs represent major problems of nursing home residents, such as cognitive loss, incontinence, and pressure ulcers (decubiti). Each RAP is "triggered" by particular MDS items or combinations of items that indicate whether the resident has, or is at risk of having, a problem in the RAP area. The RAP then provides "guidelines" for care planning, helping care planners think through possible approaches to curing the problem or at least maintaining the resident's current level. 
This article focuses primarily on those problem areas represented by the RAPs, with several additional areas not represented in the current set of RAPs. Several of the RAP domains, such as activities of daily living (ADLs) and cognition, are the subject of separate analyses described in a companion study. ${ }^{3}$ The current analysis focused on selected health conditions or problems. From a broader list of potential problems, we omitted those with extremely low prevalence (hunger or internal bleeding) or relatively lesser importance for nursing home populations (such as debris in mouth before going to bed). Out of this list, before the data analysis, we selected eight conditions to examine: dehydration, falls, decubitus, vision problems, stasis ulcer, pain, dental status, and malnutrition.

There is considerable literature extant on the prevalence of each of the eight conditions examined here, as well as on their risk factors (for dehydration, ${ }^{4}$ falls, ${ }^{5,6}$ decubitus, ${ }^{7,8}$ vision, ${ }^{4,10}$ stasis ulcer, ${ }^{11}$ pain, ${ }^{12}$ dental status, ${ }^{13,14}$ and malnutrition $\left.^{15}\right)$. In the models here, we were primarily interested in differences in the (6-month) outcomes and in the prevalence of the conditions examined. For these tests, the inclusion of risk factors would likely obscure the primary effect examined. For example, the differences in outcomes we wished to identify might be achieved by changes in practice patterns that altered risk factors.

The research reported here examines the differences in the selected conditions between pre-RAI and post-RAI cohorts of nursing home residents. We hypothesized that there would be fewer residents acquiring the selected conditions (or declining on a functional scale, such as of vision) during the 6-month follow-up period after RAI implementation, contrasted with those residents in the pre-RAI era. We also expected comparatively more residents to have conditions resolved or to improve in function in the post-RAI era. In the description that follows we examine these changes and attempt to differentiate the effects seen from other changes in nursing home practice that have occurred simultaneous to the RAI implementation.

\section{METHODS}

\section{Sample}

Data were collected for this study using a cluster sample within 10 states, in two rounds. In the first round data collection (1990), states were chosen based on four criteria: geographic location (federal region), reimbursement methodology (case mix or non-case mix), high/low Medicaid reimbursement level, and high/low staffing levels (as reported in the federal Medicare/Medicaid Automated Certification System). To increase efficiency, data collection within each state was concentrated in counties in one standard metropolitan area (MSA) and more rural counties adjacent to each MSA. Twenty-four facilities in each MSA and three rural facilities were recruited for each state (a minimum bed size of 25 was used, to assure a sufficient sample of residents). In each facility, sample sizes of $8-16$ residents were chosen, based on the size of the facility. One quarter of the sample were new admissions (between 30 and 60 days of admission), the remainder from those in the facility over 60 days. The second round of data collection, in 1993, went back to these same facilities. Three facilities had gone out of business, $10 \mathrm{de}-$ clined participation, and 1 was deemed ineligible. In total, $95 \%$ of the first-round facilities participated in the second round of data collection. A new sample of residents was drawn, using the same protocols, except that residents were selected randomly rather than stratified by length of stay. Detailed description of the data collection appears in Phillips et al. ${ }^{3}$

For each resident sampled in either round, data collection included a full MDS, completed by project-trained nurses at baseline and again, 6 months later (follow-up). As always with the MDS, assessments used multiple sources of information to identifying resident characteristics, including discussion with and observation of the resident, discussions with facility staff of all shifts, the medical record, and so forth. The same protocols were used in 1990 and 1993. Residents no longer in the facility at follow-up were tracked through all locations (hospitals, back to the same or other nursing homes, or to a private home in the community), or to death. These activities are documented in a companion study on transitions. ${ }^{16}$

In total, the sample used in this analysis was a cohort of 2,128 residents from 268 nursing homes in the first round, and second cohort of 2,088 from 254 of the same homes in the second round. Weights assure that the samples appropriately represent the study population, by adjusting for differences in the number of facilities chosen in each location and the numbers and types of residents sampled. Each cohort represents a population of more than 500 nursing homes in the 10 states, with over 60,000 residents.

\section{Model Specification:}

We used logistic regression to examine the effect of the RAI on dichotomous outcome variables. The analysis was designed to address the problem of differential exits, for an improvement such as slowed decline in the second round (post-RAI implementation) could have been achieved by having, for example, a higher mortality rate for those who had declined. The approach taken for each of the eight conditions was to examine two dichotomous outcome contrasts, all compared with the stable outcome (that the resident was in the same status with respect to the dependent variable at baseline and at follow-up). Therefore, after eliminating those who exited, we contrasted decline versus stability and improved versus stability. (A third set of models, contrasting exit and stability, are not reported here. There were no significant differences in the two cohorts in the rate of exit. ${ }^{16}$ ) Those who could not decline, because they had the condition or were at the lowest level already at baseline, were eliminated from the analysis of decline; those who could not improve were similarly eliminated from the analysis of improvement. For example, to test vision, we considered the visual level of residents at baseline and at follow-up. Regardless of initial condition at baseline, residents who left to home, hospital, or by death were classified as exiting. Residents who did not exit and were assessed at the same level at both times were considered stable. Among those who were able to decline (i.e., those with other than the most severe visual impairment at baseline), those who did not exit and at 6-month follow-up were at a less functional level on the 4-category MDS scale were considered to have declined. Those improving were identified in a similar way. This logic formed the "decline" and "improve" dichotomous variables to test (vision) outcomes. For dichotomous variables such as stasis ulcer, decline represented the incidence at the 6-month follow-up assessment among a restricted sample of those who 
were free of any stasis ulcer at baseline; improvement was the incidence of a resolution in the sample of those with an existing stasis ulcer at baseline.

\section{Outcome Variables}

Of the eight domains selected for examinationdehydration, falls, decubiti, vision problems, stasis ulcer, pain, dental status, and malnutrition-all except stasis ulcer and pain have their own RAP. Other than dental status and malnutrition, all are represented by single items on the MDS. For dental status, from six potential dental items, we focused on that describing broken, loose or carious teeth (abbreviated in this article as "poor teeth"). Other dental items were either very unlikely (e.g., inflamed gums) or more a measure of process than of condition (e.g., daily cleaning of teeth/ dentures). To represent malnutrition, we employed the body mass index (BMI). ${ }^{17}$ The $\mathrm{BMI}$, also referred to as the Quitelet Index, is the ratio of body weight to surface area, or, more particularly BMI = weight $/(\text { height })^{2}$, where weight is in kilograms and height in meters. (When using inches and pounds, as in the MDS, the right side of the equation can be multiplied by 703.1 to achieve the correct result.) The BMI is regularly used to represent nutritional level and has been considered the best simple indicator of the total amount of body fat. ${ }^{18}$ Both low and high BMI have been associated with increased mortality in many (although not necessarily nursing home) populations. ${ }^{19}$ There are no agreed standards for malnutrition for the BMI in nursing home residents. We used the threshold of the $33 \mathrm{rd}$ percentile in our data; residents with BMI below 20 were deemed malnourished (a parallel analysis, using a score of 19 -the 2.5 th percentile-showed similar results).

For outcome variables that represented dichotomies, we contrasted only the presence or absence of the condition. For the two outcome variables with multiple levels (i.e., vision and pressure ulcers), we considered any improvement or decline from the baseline level. Of the final list of variables, all had at least acceptable inter-rater reliability (kappa values in excess of $0.41 .^{20}$

\section{Independent Variables}

The primary independent variable in the logistic model was an indicator of "post-RAI cohort," which tested the hypothesis of significant outcome differences before and after RAI implementation. Covariates in the main effects model adjusted for other major characteristics of the resident which might have an effect on outcomes. These covariates included measures of cognitive performance, functionality, case-mix intensity, and, where appropriate, baseline status of the condition.

Cognitive function was measured by the MDS Cognitive Performance Scale (CPS). ${ }^{21}$ This scale has been shown to correlate well with a gold-standard clinical measure of cognitive function, the Folstein Mini-mental Status Examination, ${ }^{22}$ and its variables have very high measured interobserver reliability. ${ }^{20}$ The CPS has seven categorical levels, ranging from "intact" to "very severely impaired." The CPS was entered in the models as six indicator variables, with "intact" as the reference group.

Physical function was measured by a composite scale of MDS ADLs. ${ }^{23}$ This scale combines the ADL self-performance variables of transfer, locomotion, eating, toileting, dressing and bathing, and bladder continence. The lowest score is indicated for residents who are essentially continent and independent in the six ADL variables; on the other end of the scale, the highest value is assigned to residents who are dependent in eating and at least two other ADLs, or one ADL and totally incontinent. This scale has been shown to be negatively related to 6-month mortality and positively related with returning home. With six categorical levels, we represented this scale in our models with five dichotomous variables, with "independently functioning" as the reference category. The ADLs have some of the highest interobserver reliabilities of all MDS items. ${ }^{20}$

Case-mix intensity was measured using the case-mix index of a modified Resource Utilization Group (RUG-III) classification. The original RUG-III system categorizes residents into 44 mutually exclusive groups using primarily the type of resident and a composite measure of resident function (ADLs) ${ }^{24}$ RUG-III groups are computed using items from the MDS and a limited number of additional items (available in the MDS+ instrument used in several states that have implemented RUG-III as part of case-mix payment systems for nursing homes-they will also be available in the new MDS Version 2.0). As these additional items were not available here, we developed a "RUG-III/MDS" system without them. The RUG-III/MDS has a total of 31 groups and is nearly as powerful as the RUG-III system in explaining nursing home costs. RUG-III/MDS explains $38 \%$ of the variation in measured staff costs (weighted nursing time, for nurses and aides) in the original RUG-III derivation sample of over 7,000 residents in seven states. (For contrast, the full RUG-III system achieved $41 \%$ variance explanation of this same variable; the RUG-IIV/MDS and RUG-III systems also explain, respectively, 49.2 and $55.5 \%$ of the variance in totalnursing plus therapy-costs.) We used these same data to develop a case-mix index (CMI) for the RUG-III/MDS. Although only manifesting 31 discrete values, the CMI represents the (numeric) relative cost of each of the RUG-III/MDS groups. This CMI variable was used here as a continuous covariate to represent baseline case mix in the logistic regression.

The ADL scale and RUG-III/MDS CMI are correlated. However, as the hypothesis tests only the significance of "cohort," and as we do not attempt to interpret the coefficients of other variables in the model, this causes no analytic problems. We also did not enter any of the many possible "risk adjusters" for the eight conditions, as this could "overcorrect": facilities would likely address such risk factors as part of an appropriately performed, RAI-driven, care planning process.

For two of the outcome variables, vision and decubitus, the baseline value was entered as a covariate, to adjust for initial status. This inclusion was not necessary in models for the remaining six (dichotomous) outcome variables, as it was implicitly accomplished by restricting the sample considered. In models of improvement for dichotomous conditions, we only included cases that could improve, i.e., those that had the condition at baseline. Similarly, in models of decline, we included only cases that were free of the condition at baseline.

Additional logistic regressions were run to assure that neither other resident characteristics nor facility characteristics would explain any differences seen in the pre- and postRAI cohorts. These models included resident gender, length of stay (grouped into those staying less than 60 days versus all others) and age (grouped by decades from 65 to 8.5 years old); 
and facility characteristics including bed size $(0-120,121-$ 200 , and over 200 beds), ownership (for profit, not for profit), urban/rural, freestanding/hospital-based, staffing level, and state. Again, the goal was not to estimate these effects; rather it was to determine if their addition to the model drew explanatory power away from the variable identifying the cohorts.

Although the pre/post differences in decline and in improvement of each of the eight conditions were evaluated by testing the coefficient of the cohort indicator variable, we also developed a summary measure across all eight conditions. Decline and improvement were modeled separately. We computed a standardized score for each condition by dividing each conditions cohort coefficient by its standard deviation and summing. As there were only weak correlations among the outcome variables, we then applied the standard $z$-test (with known variance of the square root of 8 ) to each of the two statistics.

\section{Analytic Methods}

To adjust the variance of estimates for sampling effects', weights were computed based on the sampling design, and the analysis was performed using the logistic regression procedure in SUDAAN. ${ }^{25}$ Comparisons of baseline prevalences were also performed in SUDAAN, using the chi-squared statistic. Other analyses presented here for descriptive purposes used other procedures from $\mathrm{SAS}^{26}$ although again using weights to adjust for the effects of sampling design. Tests were considered significant at the .05 level. In a few instances, tests were applied to less than 100 observations in each wave; despite the power of the weighted statistical procedure, these results involve very small numbers of observations, and were considered inappropriate. With two simultaneous contrasts for each outcome considered by logistic regression, we applied the Bonferroni correction ${ }^{27}$ and applied a significance level one-half as high $(.025)$ as that traditionally applied.

\section{RESULTS}

Descriptive baseline statistics on the 4,216 residents (representing a population of 121,337 ) in the combined pre- and post-RAI samples (Table 1) show a population very similar to that seen in other studies (see Phillips et al. ${ }^{3}$ and Mor et al. ${ }^{16}$ for an analysis of the effects of the RAI implementation on hospitalization and other exit rates).

The prevalence of the eight selected health conditions at baseline ranged from $2 \%$ (dehydration) to $59 \%$ (any visual impairment) in the pre-RAI cohort (Table 2 ). In the post-RAI cohort, prevalences generally dropped, for example to 1 and $54 \%$ for these same two conditions, respectively. Of the eight conditions, two (dehydration and stasis ulcer) showed a decline in prevalence by the post-RAI round that was statistically significant. Two conditions showed an increase from pre- to post-RAI: the percentage of residents who had falls increased insignificantly, but the increase in those who had daily pain was statistically significant. Still, overall, the average RUG-III/MDS case-mix index changed only $1.7 \%$ (from 0.89 to $0.91, P=.20$ ) between the two cohorts.

Results from the weighted logistic regressions of sixmonth outcomes (decline and improvement) for the eight selected conditions are displayed in Table 3 . In particular, the coefficient, odds ratio, and significance of this coefficient are provided, along with the number of observations in both
Table 1. Descriptive Statistics of Sample Residents at Baselines

Raw Weighted

Variable*

Frequency Percent†

\begin{tabular}{|c|c|c|}
\hline \multicolumn{3}{|l|}{ Cohort } \\
\hline Pre-RAI & 2128 & \\
\hline Post-RAl & 2088 & \\
\hline \multicolumn{3}{|l|}{ Cognitive performance $\neq$} \\
\hline Intact/borderline intact & 1296 & 30.9 \\
\hline $\begin{array}{l}\text { Moderate, moderately severe, and } \\
\text { severe impairment }\end{array}$ & 1507 & 34.6 \\
\hline \multirow{2}{*}{\multicolumn{3}{|c|}{ ADL§ }} \\
\hline & & \\
\hline $\begin{array}{l}\text { Independent/supervision/limited } \\
\text { assistance }\end{array}$ & 770 & 19.0 \\
\hline Limited/extensive assistance & 1279 & 29.6 \\
\hline Some and total dependency & 2150 & 51.4 \\
\hline \multicolumn{3}{|l|}{ Age } \\
\hline$<65$ years & 308 & 6.9 \\
\hline 65 to $<75$ years & 513 & 11.5 \\
\hline 75 to $<85$ years & 1384 & 33.2 \\
\hline 85 years or older & 2029 & 48.4 \\
\hline \multicolumn{3}{|l|}{ Gender } \\
\hline Male & 1026 & 23.3 \\
\hline Female & 3215 & 76.7 \\
\hline \multicolumn{3}{|l|}{ Length of stay } \\
\hline$<60$ days & $\begin{array}{r}751 \\
3508\end{array}$ & 9.5 \\
\hline
\end{tabular}

Combined pre- and post-RAl cohorts.

* Levels of the C.PS and ADL variables have been combined for clarity; the full range of levels were used in other analyses.

† Using sampling weights to adjust to population prevalences ( $n=121,000$ ).

\# Measured by the Cognitive Performance Scale, described in Morris et al. ${ }^{21}$

$\$$ See definition of ADL scale in Mor et al. ${ }^{23}$

waves, combined, that are included in the analysis. As discussed earlier, several models involved very small numbers of observations (for improvement: dehydration, stasis ulcer, and poor teeth), and are not considered. Taking dehydration as an example, only 60 residents ( $3 \%$ of total) had dehydration at baseline in the pre-RAI sample, and only $22(1 \%)$ in the post-RAI wave. Within these groups, only 2 (pre-) and 4 (post-RAI) nonexiting residents showed improvements.

In models contrasting 6-month rate of decline in the eight selected health conditions, two conditions-malnutrition and vision problems-showed statistically significant reductions between the two cohorts. For malnutrition, after adjusting for other covariates, the post-RAI cohort was only $50 \%$ as likely to have a decline from baseline compared to the 1990 cohort; for vision, 44\%. Although not significantly different from zero, we still note that seven of the eight conditions exhibited decline and the summary score for the eight decline coefficients is -6.62 and highly significant at $P<.0001$.

At the same time, six of the eight conditions showed reduced improvement rates for the post-RAI cohort, although two of these involved very small samples. For the two conditions with significant results for decline-malnutrition and vision problems-improvement rates were also significantly reduced, as was the decline rate for pain. For the eight conditions the summary statistic was -5.23 and significant at 
Table 2. Prevalence of Eight Conditions at Baseline, by Cohort

\begin{tabular}{|c|c|c|c|c|c|}
\hline \multirow[b]{2}{*}{ Health condition } & \multicolumn{2}{|c|}{ Pre-RAI } & \multicolumn{2}{|c|}{ Post-RAl } & \multirow[b]{2}{*}{ Significance $†$} \\
\hline & $\%$ & $\mathrm{n}^{*}$ & $\%$ & $n^{*}$ & \\
\hline Dehydration & 2.0 & 1,264 & 1.0 & 558 & .01 \\
\hline Fall in 30 days & 10.5 & 6,597 & 10.6 & 6,178 & .97 \\
\hline Malnutrition (BMI < 20) & 32.1 & 17,830 & 30.9 & 17,597 & .47 \\
\hline Decubitus: & & & & & .10 \\
\hline Stage 1 & 5.0 & 3,111 & 3.8 & 2,228 & \\
\hline Stage 2 & 6.4 & 4,032 & 6.0 & 3,538 & \\
\hline Stage 3 & 1.7 & 1,086 & 2.0 & 1,174 & \\
\hline Stage 4 & 0.9 & 587 & 0.5 & 280 & \\
\hline Vision: & & & & & .17 \\
\hline Impaired & 35.4 & 20,957 & 35.4 & 20,133 & \\
\hline Highly & 17.2 & 10,211 & 12.8 & 7,342 & \\
\hline Severely & 6.7 & 3,958 & 5.7 & 3,245 & \\
\hline Stasis ulcer & 4.5 & 2,791 & 3.0 & 1,750 & .02 \\
\hline Daily pain & 13.4 & 8,414 & 17.0 & 9,931 & .01 \\
\hline Poor teeth & 4.6 & 2,905 & 3.9 & 2,282 & .31 \\
\hline
\end{tabular}

- Weighted frequency.

† Test for differences in prevalence at baseline was performed using the chi-squared statistical test within SUDAAN, using weights to adjust for sampling bias. Significance is achieved at $\alpha=5 \%$.

Table 3. Coefficient of Cohort (Pre- vs Post-RAI) in Two Logistic Regression Models, for Each of Eight Conditions

\begin{tabular}{|c|c|c|c|c|c|c|c|c|}
\hline \multirow{2}{*}{$\begin{array}{l}\text { Health } \\
\text { Condition }\end{array}$} & \multicolumn{4}{|c|}{ Decline } & \multicolumn{4}{|c|}{ Improvement } \\
\hline & Coefficient & $\mathrm{OR}^{*}$ & Significance $†$ & $N$ & Coefficient & $\mathrm{OR}^{*}$ & Significance† & $N$ \\
\hline Dehydration & 0.08 & 1.08 & .82 & 3326 & -2.81 & 0.06 & $.008 \dagger$ & $55 \ddagger$ \\
\hline Falls & -0.24 & 0.79 & .10 & 3005 & 0.18 & 1.20 & .51 & 382 \\
\hline Malnutrition & -0.69 & 0.50 & $<.0001 \dagger$ & 2292 & -0.56 & 0.57 & $.0003 \dagger$ & 1102 \\
\hline Decubitus & -0.22 & 0.81 & .13 & 3364 & -0.10 & 0.90 & .70 & 478 \\
\hline Vision problems & -0.82 & 0.44 & $<.0001 \dagger$ & 3094 & -0.50 & 0.60 & $<.0001 \dagger$ & 1934 \\
\hline Stasis ulcer & -0.35 & 0.70 & .21 & 3262 & 0.58 & 1.79 & .25 & $125 \ddagger$ \\
\hline Pain & -0.06 & 0.94 & .67 & 2867 & -0.64 & 0.53 & $.005 \dagger$ & 519 \\
\hline Poor teeth & -0.26 & 0.77 & .29 & 3241 & -1.50 & 0.22 & $.005 \dagger$ & $147 \ddagger$ \\
\hline
\end{tabular}

Dependent variables are the presence of either decline (improve), out of the population which has not exited and is able to decline (improve). Independent variables in equation are CPS, ADL scale, RUG-III/MDS CMI, and cohort. For decubitus and vision models, baseline values are also included as independent variables.

- Odds ratio from logistic regression.

$\dagger$ Indicates significant effect of cohort at $\alpha=5 \%$ (adjusted to $\alpha=2.5 \%$, with Bonferroni correction).

$\ddagger$ Test involves less than $n=100$ in each wave.

$P<.0001$ (the score dropping the three conditions with small numbers of observations was similarly significant).

Secondary logistic models were fitted, using additional variables to represent resident characteristics (age, gender, and length of stay) or facility characteristics (size, ownership, urban/rural, free-standing/hospital-based, staffing level, and state). These models did not demonstrate any consistent indication that the results described above would change.

That there were post-RAI reductions in the rates of decline and improvement for the same variable-malnutrition and vision-provides potentially confusing findings: with both decline and improvement outcomes reduced, it is unclear what was the net effect.

Both to untangle and compound these effects, we consider here the example of outcomes in malnutrition. In this work, we simplify our models from the multivariate logistic results and consider only the primary effects: (weighted) prevalences and 6-month transition probabilities. Therefore, these results augment and explain, but do not replace, the confirmatory results of the logistic regression.

Comparing the cohorts at the baselines, there were fewer residents in the post-RAI cohort who were malnourished $($ BMI $<20)$. Figure 1 shows the 6-month outcomes for malnourished residents, with the percentages displayed for the total baseline sample (malnourished and nourished), i.e., out of $n=2128$ or $n=2088$ for the pre- and post-RAI cohorts, respectively. We see here slightly different rates of improvement in nutrition for malnourished residents who did not exit $(6.5 \%$ in the pre-RAI round, compared with $5.0 \%$ in the post-RAI round). Slightly more nonexiting residents also remained malnourished (18.4 vs $19.6 \%$, respectively). On the other hand, for the more numerous residents with adequate BMI at baseline, a greater percentage 6 months later remained nourished in the post-RAI cohort $(50.2$ and $53.9 \%$, in the pre- and post-RAI cohorts, respectively), and fewer declined (6.6 vs $5.3 \%$, respectively) (Figure 2 ). If we consider 


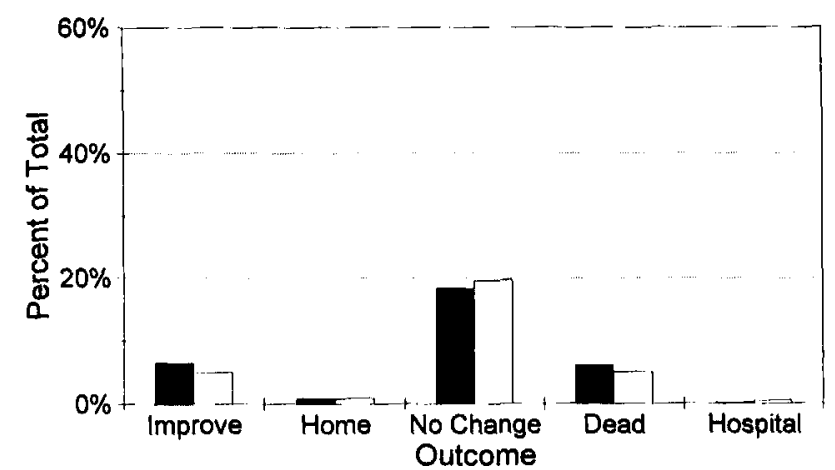

Figure 1. Six-month changes in nutrition for persons with low BMI $(<20)$. $\square$, Pre-RAI; $\square$, post-RAI.

adequate BMI and discharge to home as positive outcomes, then a sum of these statistics shows that $59.5 \%$ of the all residents in the pre-RAI round had good outcomes, with this percentage increasing to $61.6 \%$ in the post-RAI round. Alternately stated, although the rate of improvement was reduced in the post-RAI cohort, its effect was more than compensated by the reduced rate of decline for the larger segment of the population (those with adequate BMI).

Similar accumulations can be performed for vision. The results are summarized in Figure 3 that displays the strict averages of the vision scale, which ranges from 0 (adequate vision) to 3 (severely impaired vision). (This scale has not been proven to be numeric, so any averages of the values are solely for the purposes of display.) We see again that at baseline, the average vision of residents was better in the post-RAI cohort. Also, although vision declined in both rounds (i.e., the average vision score increased) from baseline to follow-up, the decline was slower in the post-RAI cohort.

\section{DISCUSSION}

Of eight health conditions representing poorer health status, two - dehydration and stasis ulcer - had significantly lower prevalence after the implementation of the RAI (in 1993) compared with 1990. This occurred within samples drawn carefully from the same facilities and with the same sampling methodologies. In addition, for one condition, daily pain, there was an increase in prevalence. Overall, there was essentially no difference in case-mix (as seen in the RUG-III/ MDS case mix index) between these two (baseline) samples, even when tested with the considerable power generated by the sampling design.

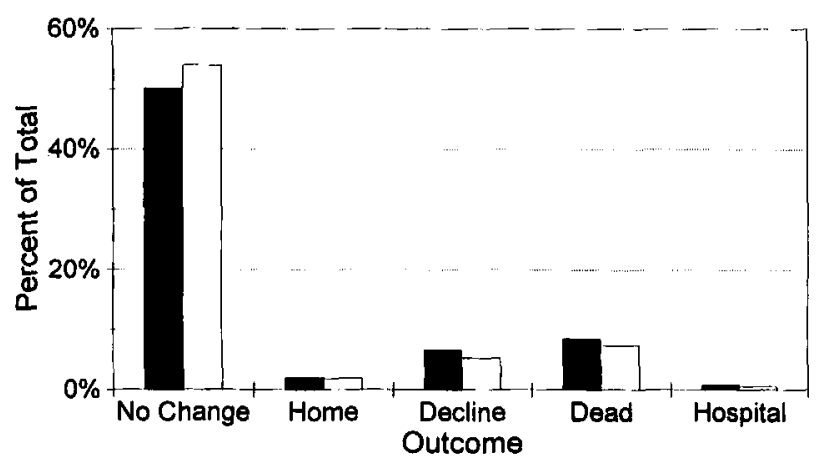

Figure 2. Six-month changes in nutrition for persons with adequate BMI ( $\geq 20)$. $\mathbf{a}$, Pre-RAI; $\square$, post-RAI.



Figure 3. Changes in average vision score for pre- and post-RAI samples.

Perhaps more significantly, two out of these eight conditions demonstrated fewer residents declining after implementation: nutrition and vision. These same two conditions had significantly reduced rates of 6 -month improvement. However, the improvements occurred for a more restricted subpopulation (those with the condition at baseline), and the net was an overall reduction in the rate of decline for all residents. Only one other condition demonstrated significant change in other than a very limited population: the resolution of severe pain was also lower in 1993. Overall, the combined eight conditions showed reductions in both the rates of decline and improvement.

Of the four conditions for which there are significant declines in prevalence or outcomes (dehydration, stasis ulcer, nutrition, and vision) three are specifically addressed in the care planning guidelines incorporated the RAI system (all except stasis ulcer, although there is a RAP for decubitus ulcer). Pain, the only other condition with a significant result-an increase in baseline prevalence and a decline in the improvement rate- has no RAP.

These findings provide partial support for our hypothesis that there were declines in the prevalence and 6-month outcomes of the eight selected health conditions over the period from 1990 to 1993. Although our models of outcomes are not strictly measures of incidence, for residents could have had a problem condition that was resolved within the 6-month window, it can be expected that they correlate highly with incidence. Although the actual differences seen range from small to moderate, they are applied to virtually all residents in US nursing homes; for example, a decline in dehydration prevalence from 2.02 to $0.95 \%$, a decline of over $50 \%$, means that there are in the US 18,000 additional residents without this condition and a system without the cost of its care.

It remains at issue whether the detected differences can be attributed to the RAI, and are not just the result of other temporal changes in nursing homes over this period. To gain credibility, there should be evidence of a causal path to the differences seen. We examine this path here. First, three out of the five conditions with significant findings are addressed by RAP areas. For example, for dehydration - an acute condition with potential fatal consequences if not addressed-the RAP guides not only the better detection of dehydration but also the identification of risk factors (infections, fever, poly- 
uria; use of laxatives, enemas, or diuretics; and systolic/ diastolic blood pressure drop upon standing). The nutrition RAP addresses physical problems in consuming food (e.g., swallowing problems or the lack of adaptive devices) but also problems to be reviewed for causal links (e.g., mental problems regarding eating, such a fear of poisoning). Vision guidelines address not only the availability, use, and appropriateness of glasses, but also the appropriate use of eye medications (e.g., to control glaucoma) and environmental modifications to help cope with visual problems. ${ }^{2}$ Although stasis ulcer was not directly addressed in a RAP, some similar material was covered in the RAP for pressure sores (and the drop in prevalence of decubiti was close to statistical significance). Second, we know from companion research that these same three conditions are significantly more likely addressed in a care plan in 1993 compared with $1990^{28}$; care planning for pain was not addressed in our study.

After this, however, the causal path becomes more obscure, for we do not have objective data on whether care plans were well designed nor if they were enacted. Other data indicate that staff perceive that the RAI helped them improve residents quality of care and we have parallel results for measures of process ${ }^{28}$ and outcome $e^{3}$ appearing in companion studies. At the same time, many of the other challenges to a hypothesis of causality - such as instrument effect-can be dismissed (see Phillips et al. ${ }^{3}$ ). For example, we have controlled well for other contaminations in the study by using the same facilities, the same nurse assessors, and so forth. However, there remains, as always, the possibility that protocols were not fully effective and, for example, the presence of the MDS in the nursing homes' medical records influenced the assessments in the post-RAI data collection. In most cases, however, such contamination would have the effect of reducing the effects seen here, either directly, or through increased random "noise."

Clearly the observed changes are part of more general positive changes that have occurred in nursing homes as a result of the multiple facets of the OBRA-87. But assessment and care planning have long been identified as the critical core of such improvements, as early as in the report of the Institute of Medicine ${ }^{29}$ that engendered OBRA-87. Although the RAI certainly cannot take sole credit for any detected changes in nursing homes, it has clearly been an integral part.

Along with these positive findings, we also report a negative result: that the rate of improvement declined in the post-RAI round. There is a variety of possible reasons for this result. Although we have performed extensive tests and reviews of our sampling and data collection methodology, it is always a possibility that the result is caused by some methodological contamination. A second and potentially more likely explanation is that the RAI has increased the stability of residents' health status, decreasing the cyclic incidence and resolution of problem conditions. Under this scenario, there will be lower rates of improvement because there are fewer residents with the condition or with the associated causal factors. A third hypothesis, and the one we feel is most likely, relates to the allocation of limited nursing home resources. The numerous nursing home reforms recently put in place, including the RAI, have focused attention on particular problems in the industry, including decubiti and the use of psychotropic drugs and physical restraints. With fixed and limited resources, staff have worked hard to prevent these problems, for they would be very visible failures. In turn, this effort may have taken resources and energy away from the relatively harder problem of resolving health problems that already had occurred. It is not possible to know now which of these three explanations (or others) represents the current situation.

The mixture of findings-positive and negativeencourages a careful view of the limitations of this study. From a methodological view, the study would have been strengthened if we had "gold standard" determinations of the several medical conditions considered, for any variability in the assessment by our research nurses adds unwanted "noise" to the analysis. As well, examining conditions with higher prevalence would have been more likely to lead to statistical findings by reduced variability. More importantly, as we have already discussed, we are unable to document completely the causal link between the RAI implementation and outcomes; thus, although our results are more than suggestive, we cannot make direct attribution to the RAI of the manifested changes in outcomes.

This evaluation set out to determine if the implementation of the RAI had caused differences in the care provided to this nation's nursing home population. Overall, the analysis reported here provides only one piece of an answer. For several specific health problems and conditions, prevalence and the rate at which residents decline have been reduced. These positive findings seem to be associated with the presence of the RAPs, thus encouraging all facilities to employ them to their fullest extent, and driving us to develop a more comprehensive set of RAP, for example to address problems not only of pain and stasis ulcer, but also foot care, discharge planning, and so forth. From the identification of improvement we need to draw understanding of how that improvement was achieved. In this way, we can go beyond current regulatory strategies that penalize poor care and identify and replicate models of good practice.

\section{REFERENCES}

1. Morris JN, Hawes C, Fries BE et al. Designing the National Resident Assessment Instrument for Nursing Facilities. Gerontologist 1990;30:293-307.

2. Morris JN, Hawes C, Murphy K et al. Long-Term Care Resident Assessment Instrument Training Manual. Natick, MA: Eliot Press, 1991.

3. Phillips CD, Morris JN, Hawes C et al. Association of the Resident Assessment Instrument (RAI) with changes in function, cognition, and psychosocial status. J Am Geriatr Soc, 1997;45:986-993.

4. Lavizzo-Mowrey R, Johnson J, Stolley P. Risk factors for dehydration among elderly nursing home residents. J Am Geriatr Soc 1988;36:213-218.

5. Tinetti ME, Speechley M. Prevention of falls among the elderly. N Engl J Med 1989;320:1055-1059.

6. Tinetti ME, Speechley M, Ginter SF. Risk factors for falls among elderly persons living in the community. N Engl J Med 1988;319:1701-1707.

7. Spoelhof GD, Ide K. Pressure ulcers in nursing home patients. Am Fam Phys 1993;47:1207-1215.

8. Brandeis GH, Morris JN, Nash DJ et al. The epidemiology and natural history of pressure ulcers in elderly nursing home residents. JAMA 1990;264:2905-2909.

9. Horowitz A. Vision impairment and functional disability among nursing home residents. Gerontologist 1994;34:316-323.

10. Marx MS, Werner P, Cohen-Mansfield J et al. The relationship between low vision and performance of activities of daily living in nursing home residents. J Am Geriatr Soc 1992;40:1018-1020.

11. Goldman MP, Fronek A, eds. The Alexander House Group. Consensus paper on venous leg ulcer. J Dermatol Surg Oncol 1992;18:592-602.

12. Ferrell BA, Ferrell BR, Osterweil D. Pain in the nursing home. J Am Geriatr Soc 1990;38:409-414.

13. Weyant RJ, Jones JA, Hobbins $M$ et al. Oral health status of a long-termcare, veteran population. Community Dent Oral Epidemiol 1993;21:227233. 
14. Strayer MS, Ibrahim MF. Dental treatment needs of homebound and nursing home patients. Community Dent Oral Epidemiol 1991;19:176-177.

15. Keller HH. Malnutrition in institutionalized elderly. J Am Geriatr Soc 1993;41:1212-1218.

16. Mor V, Intrator $\mathrm{O}$, Hiris J et al. Impact of the MDS on changes in nursing home discharge rates and destinations. J Am Geriatr Soc 1997;45:1002-1010.

17. Chumlea W, Roche AF, Mukherjee D. Nutritional assessment of the elderly through anthropometry, 2nd Ed. Columbus, OH: Ross Laboratories, 1987.

18. Chumlea W, Roche AF, and Mukherjee D. Some anthropometric indices of body composition for elderly adults. J Gerontol 1986;41:36-39.

19. Andres R, Elahi D, Tobin JD et al. Impact of age on weight goals. Ann Int Med 1985;103:1030-1033.

20. Hawes C, Morris JN, Phillips CD et al. Reliability estimates for the Minimum Data Set for Nursing Home Resident Assessment and Care Screening (MDS). Gerontologist 1995;35:172-178.

21. Morris JN, Fries BE, Mehr DR et al. MDS Cognitive Performance Scale. J Gerontol Med Sci 1994;49:M174-182.
22. Folstein MF, Folstein SE, McHugh PR. "Mini-mental state." A practical method for grading the cognitive state of patients for the clinician. J Psychiatr Res 1975;12:189-198.

23. Mor V, Branco K, Fleishman J et al, The structure of social engagement among nursing home residents. J Gerontol Psychol Sci 1995;50B:P1-8.

24. Fries BE, Schneider D, Foley WJ et al. Refining a case-mix measure for nursing homes: Resource Utilization Groups (RUG-III). Med Care 1994;32:66868.5.

25. Survey Data Analysis Software, Version 6.34. Research Triangle Park, NC: Research Triangle Institute, 199.3.

26. SAS Procedure Guide. Version 6, 3rd Ed. Cary, NC: SAS Institute, Inc., 1990.

27. Neter J, Wasserman W, Kutner MH. Applied Statistical Models, 2nd Ed Homewood, IL: Irwin, 1985.

28. Hawes $C$, Mor V, Philips CD et al. The OBRA-87 nursing home regulations and implementation of the Resident Assessment Instrument: effects on process quality. J Am Geriatr Soc, 1997;45:977-985.

29. Committee on Nursing Home Regulation. Improving the Quality of Care in Nursing Homes. Washington, DC: Institute of Medicine, 1986. 